\title{
Modeling acoustic waves with paraxial extrapolators
}

\author{
R. W. Graves* and R. W. Clayton*
}

\section{ABSTRACT}

Modeling by paraxial extrapolators is applicable to wave-propagation problems in which most of the energy is traveling within a restricted angular cone about a principal axis of the problem. Using this technique, frequency-domain finite-difference solutions accurate for propagation angles out to $60^{\circ}$ are readily generated for both two-dimensional (2-D) and three-dimensional (3-D) models. Solutions for 3-D problems are computed by applying the 2-D paraxial operators twice, once along the $x$-axis and once along the $y$-axis, at each extrapolation step. The azimuthal anisotropy inherent to this splitting technique is essentially eliminated by adding a phase-correction operator to the extrapolation system. For heterogeneous models, scattering effects are incorporated by determining transmission and reflection coefficients at structural boundaries within the media. The direct forwardscattered waves are modeled with a single pass of the extrapolation operator in the paraxial direction for each frequency. The first-order backscattered energy is then modeled by extrapolation (in the opposite direction) of the reflected field determined on the first pass. Higher order scattering can be included by sweeping through the model with more passes.

The chief advantages of the paraxial approach are (1) active storage is reduced by one dimension compared to solutions which must track both forwardscattered and backscattered waves simultaneously; thus, realistic 3-D problems can fit on today's computers, (2) the decomposition in frequency allows the technique to be implemented on highly parallel machines, (3) attenuation can be modeled as an arbitrary function of frequency, and (4) only a small number of frequencies are needed to produce movie-like time slices.

\section{INTRODUCTION}

Several numerical techniques have been developed for simulating wave motions in complex media. The most general of these are the finite-difference (FD) method (Kelly et al., 1976), the finite-element (FE) method (Marfurt, 1984), and the pseudospectral (PS) method (Kosloff and Baysal, 1982). In their heterogeneous formulations, these techniques are capable of complete wave solutions for arbitrary models. The chief shortcoming of these numerical simulations is that for 3-D and large 2-D problems their computational requirements overwhelm the capabilities of most computer hardware currently available. The use of supercomputers (Reshef et al., 1988a, b) or massively parallel computers (Fricke, 1988) may provide a way around this problem; however, even with this technology, the implementation of these methods is still restricted by the availability of accessible computer memory.

To address this problem, we present an approximate numerical technique that is based on one-way paraxial extrapolators. The use of paraxial extrapolators as a means of seismic wave propagation was first introduced by Claerbout (1970). Since then, this technique has been extensively developed for the migration of seismic reflection data (e.g., Claerbout, 1985). Other applications of the extrapolators include their adaptation for use as absorbing boundary conditions in FD simulations (Clayton and Engquist, 1977). The paraxial operators correctly model waves traveling within an angular cone centered about a particular axis of the problem. For example, surface waves can be modeled with horizontal extrapolators, while precritical reflections can be modeled with vertical extrapolation. These operators are usually referred to by the extent of their angular accuracy. 
The most commonly known paraxial systems are the $15^{\circ}$ and $45^{\circ}$ approximations. However, an operator which is accurate to $60^{\circ}$ can be obtained simply by modifying the coefficients in the $45^{\circ}$ approximation.

To set up the paraxial system, we first arrange the equations of motion into a first-order extrapolation system. In doing this, we effectively reduce the computer memory requirements needed to calculate the solution by one spatial dimension compared to the complete methods. This reduction enables us to generate solutions to 3-D problems simply by extrapolating the wave field through the model on a 2-D plane with the end result that storage requirements are rarely a limiting factor when using this technique on large 2-D or 3-D problems.

In the next section, we derive the general form of the extrapolation system for 3-D problems. This derivation is followed by a discussion of the paraxial approximation and the implementation of the resulting numerical system, including the incorporation of sources and scattering effects, for both 2-D and 3-D models. Our formulation is developed in the temporal frequency domain; some advantages of using this domain are also discussed. Finally, we present some examples of the application of our technique to various seismic problems.

\section{THE 3-D EXTRAPOLATION SYSTEM}

In this section the acoustic (scalar) extrapolators are derived for a general 3-D medium. The extrapolators are based on the coupled first-order equations

$$
\begin{aligned}
& \rho \omega^{2} \mathbf{u}=\boldsymbol{\nabla} \boldsymbol{P}+\mathbf{f}_{F} \\
& \boldsymbol{P}=-K \boldsymbol{\nabla} \cdot \mathbf{u}+f_{P},
\end{aligned}
$$

where $P(x, y, z, \omega)$ is the pressure, $\mathbf{u}=(u, v, w)^{T}$ is the displacement, $\rho(x, y, z)$ is the density, $K(x, y, z)$ is the bulk modulus (compressibility), and $\omega$ is the frequency. The terms $\mathbf{f}_{F}=\left(f_{x}, f_{y}, f_{z}\right)^{T}$ and $f_{P}$ are the force and pressure sources of the system, respectively. Equations (1) can be recast into the first-order extrapolation system

$$
\partial_{z}\left[\begin{array}{l}
P \\
w^{\prime}
\end{array}\right]=\mathbf{A}\left[\begin{array}{l}
P \\
w^{\prime}
\end{array}\right]+\mathbf{f}
$$

where

$$
\mathbf{f}=\left[-f_{z} \cdot \frac{1}{\rho v^{2}} f_{P}-\frac{1}{\omega^{2}}\left(\partial_{x} \frac{1}{\rho} f_{x}+\partial_{y} \frac{1}{\rho} f_{y}\right)\right]^{T}
$$

is the source vector and $\mathbf{A}$ is the matrix

$$
\mathbf{A}=\left[\begin{array}{cc}
0 & \\
-\frac{1}{\rho \omega^{2}} \alpha^{2} & 0
\end{array}\right]
$$

with $\alpha$ given by the pseudodifferential operator

$$
\alpha=\left(\frac{\omega^{2}}{v^{2}}+\rho \partial_{x} \frac{1}{\rho} \partial_{x}+\rho \partial_{y} \frac{1}{\rho} \partial_{y}\right)^{1 / 2},
$$

where $v^{2}=K / \rho$. The symbols $\partial_{x}, \partial_{y}$, and $\partial_{z}$ are used as shorthand representations of the differential operators $\partial / \partial x$, $\partial / \partial y$, and $\partial / \partial z$.
Using equation (2) to extrapolate wave fields through heterogeneous media presents a problem because this system is complete and propagates both forward and backscattered wave fields simultaneously. Thus, in order to start the extrapolation process, we need to specify the entire wave field (forward and backscattered energy) for all time along one boundary of the model. This is problematic in modeling exercises, since we generally have a priori information only about the source and not about energy which has propagated through the region to be modeled. For this reason, we need to find an alternative formulation of the extrapolation system.

Our approach is to start from equation (2) and form a new set of decoupled paraxial extrapolators. The advantage of this method is that the propagation aspects of the new system are well understood and all that remains is to incorporate the effects of scattering.

\section{Decoupling the first-order system}

To form a set of paraxial extrapolators for equation (2), the A matrix is decomposed into its eigenvalue and eigenvector representation. That is,

$$
\mathbf{A}=\underline{\mathbf{E}} \mathbf{\Lambda} \underline{\mathbf{E}}^{-1}
$$

where

$$
\begin{aligned}
& \boldsymbol{\Lambda}=\left[\begin{array}{cc}
i \alpha & 0 \\
0 & -i \alpha
\end{array}\right], \\
& \underline{\mathbf{E}}=\left[\begin{array}{cc}
1 & 1 \\
\frac{i}{\rho \omega^{2}} \alpha & \frac{-i}{\rho \omega^{2}} \alpha
\end{array}\right],
\end{aligned}
$$

and

$$
\underline{\mathbf{E}}^{-1}=\frac{1}{2}\left[\begin{array}{cc}
1 & \frac{-i}{\alpha} \rho \omega^{2} \\
1 & \frac{i}{\alpha} \rho \omega^{2}
\end{array}\right] .
$$

We now define a new solution vector

$$
\left[\begin{array}{l}
P_{f} \\
P_{b}
\end{array}\right]=\underline{\mathbf{E}}^{-1}\left[\begin{array}{l}
P \\
w
\end{array}\right]
$$

where $P=P_{f}+P_{b}$. The subscripts $f$ and $b$ refer to the forward-scattered and backscattered portions of the pressure field, respectively. By convention, we have chosen the forward-scattered pressure field $P_{f}$ to represent energy which is propagating in the positive $z$ direction. Using equations (6) and (8) in equation (2) transforms the extrapolation system into

$$
\partial_{z} \mathbf{E}\left[\begin{array}{l}
P_{f} \\
P_{b}
\end{array}\right]=\mathbf{E} \boldsymbol{\Lambda}\left[\begin{array}{l}
P_{f} \\
P_{b}
\end{array}\right]+\mathbf{f} .
$$

Differentiating through the left side, premultiplying by $\underline{\mathbf{E}}^{-1}$, and defining

$$
\mathbf{f}_{E}=\underline{\mathbf{E}}^{-1} \mathbf{f} \text { and } \mathbf{S}=-\underline{\mathbf{E}}^{-1} \underline{\sim}_{z},
$$

where $\mathbf{E}_{z}=\partial \mathbf{E} / \partial z$, we have 


$$
\partial_{z}\left(\begin{array}{l}
P_{f} \\
P_{b}
\end{array}\right)=\underline{\Lambda}\left(\begin{array}{l}
P_{f} \\
P_{b}
\end{array}\right)+\underline{\mathbf{S}}\left(\begin{array}{c}
P_{f} \\
P_{b}
\end{array}\right)+\mathbf{f}_{E} .
$$

The propagation aspects of the forward and backscattered waves of this system are now decoupled. They remained coupled through the scattering matrix $\mathbf{S}$, which is nonzero only at points where the medium changes. We will treat the scattering as if it were a pseudosource. First, however, approximations for the $\underline{\Lambda}$ operator are presented.

\section{THE PROPAGATION MATRIX}

If we neglect for a moment the real sources of equation (10) and allow the media to vary only as a function of $x$ and $y$, but not of $z(S=0)$, then the problem reduces to solving the decoupled system

$$
\partial_{z}\left[\begin{array}{c}
P_{f} \\
P_{b}
\end{array}\right]=\left[\begin{array}{cc}
i \alpha & 0 \\
0 & -i \alpha
\end{array}\right]\left[\begin{array}{l}
P_{f} \\
P_{b}
\end{array}\right],
$$

where $v=v(x, y)$ and $\rho=\rho(x, y)$. The operator $\alpha$, while symbolically represented by the pseudodifferential operator of equation (5), needs to be placed in a rational form for actual use. In order to accomplish this, let us consider only the forward-scattered portion of equation (11),

$$
\partial_{z} P_{f}=i \alpha P_{f} .
$$

Factoring out the wave field $P_{f}$, we obtain the following relation:

$$
\dot{\partial}_{z}=i \alpha .
$$

Substituting the expression for $\alpha$ from equation (5) and writing this equation symbolically, we have

$$
D_{z}=\left[1+\left(D_{x}^{2}+D_{y}^{2}\right)\right]^{1 / 2},
$$

where

$$
\begin{aligned}
& D_{x}^{2}=\frac{v^{2}}{\omega^{2}} \rho \partial_{x} \frac{1}{\rho} \partial_{x}, \\
& D_{y}^{2}=\frac{v^{2}}{\omega^{2}} \rho \partial_{y} \frac{1}{\rho} \partial_{y},
\end{aligned}
$$

and

$$
D_{z}=\frac{v}{\omega} \partial_{z}
$$

Equation (14) represents the exact one-way propagation operator.

Treating the symbols $D_{x}^{2}$ and $D_{y}^{2}$ as numerical representations of the corresponding differential operators, we expand equation (14) with a continued-fraction representation and obtain the general second-order approximation

$$
D_{z} \approx i\left[1+B\left(D_{x}^{2}+D_{y}^{2}\right)\right]^{-1}\left[1+A\left(D_{x}^{2}+D_{y}^{2}\right)\right]
$$

(Claerbout, 1985, p. 83). Here $A$ and $B$ are constant coefficients which can be chosen to maximize the accuracy of equation (15) over a given range of propagation angles (e.g., $A=1 / 2, B=0$ is the $15^{\circ}$ approximation, $A=3 / 4, B=1 / 4$ is the $45^{\circ}$ approximation, and $A=0.855, B=0.355$ is the $60^{\circ}$ approximation) (Halpern and Trefethen, 1988).
Expressions like relation (15) are usually derived by assuming a homogeneous medium and performing the expansion in the wavenumber-frequency domain. For our purposes, however, we must take care to ensure that relation (15) provides a reasonable approximation to the exact operator for laterally varying media as well. Clearly, this expression reduces to the proper formulation for the homogeneous case; furthermore, since it is a local operator, the expression is also appropriate within locally homogeneous regions of heterogeneous models. The main question remaining then is how well this operator matches the correct reflection and transmission coefficients as the wave field is propagated across a lateral boundary between different types of media. Answering this question directly is difficult because the exact operator does not have a simple analytic representation. However, we can obtain an idea of the order of accuracy by comparing the square of the exact operator with the square of the approximate operator. Here, the square of an operator is defined as the operator applied to itself.

From equation (14), we have for the exact operator (recalling that $z$ is not a function of $z$ )

$$
D_{z}^{2}=\frac{r^{\prime 2}}{\omega^{2}} \partial_{z z}=1+\left(D_{x}^{2}+D_{y}^{2}\right) .
$$

Now using the expansion

$$
\left[1+B\left(D_{x}^{2}+D_{y}^{2}\right)\right]^{-1}=\left[1-B\left(D_{x}^{2}+D_{y}^{2}\right)+B^{2}\left(D_{x}^{2}+D_{y}^{2}\right)^{2}-\cdots\right],
$$

we obtain for the approximate operator

$$
D_{z}^{2} \approx 1+2(A-B)\left(D_{x}^{2}+D_{y}^{2}\right)+O\left[\left(D_{x}^{2}+D_{y}^{2}\right)^{2}\right] .
$$

As long as $A-B=\frac{1}{2}$, the approximation is accurate to $O\left[\left(D_{x}^{2}+D_{y}^{2}\right)^{2}\right]$. In most cases, the squared term is quite small for propagation angles within the range of validity of the operator as determined by the coefficients $A$ and $B$, behavior that is demonstrated below by example.

We now substitute relation (15) into equation (12) to obtain the paraxial wave equation

$$
\frac{\tau^{\prime}}{\omega} \dot{\partial}_{z} P_{f}=i\left[1+B\left(D_{x}^{2}+D_{y}^{2}\right)\right]^{-1}\left[1+A\left(D_{x}^{2}+D_{y}^{2}\right)\right] P_{f} .
$$

The corresponding Crank-Nicolson difference equation is given by

$$
\begin{aligned}
\zeta^{-1}\left(P_{f}^{n+1}-P_{f}^{n}\right)= & i\left[1+B\left(D_{x}^{2}+D_{y}^{2}\right)\right]^{-1} \\
& \times\left[1+A\left(D_{x}^{2}+D_{y}^{2}\right)\right]\left(P_{f}^{n+1}+P_{f}^{n}\right)
\end{aligned}
$$

where $\zeta=\omega \Delta z / 2 v$ and $P_{f}^{n}=P_{f}\left(x, y, z=z_{n}, \omega\right)$. We want to solve this equation for the wave field $P_{f}^{n+1}$; however, since the operators in equation (18) do not commute, we must take care to preserve the correct operator ordering. Performing the appropriate algebra, we obtain

$$
\begin{aligned}
& \left\{1+\frac{1}{y} B\left(D_{x}^{2}+D_{y}^{2}\right) v-i \zeta\left[1+A\left(D_{x}^{2}+D_{y}^{2}\right)\right]\right\} P_{f}^{n+1} \\
& \quad=\left\{1+\frac{1}{i} B\left(D_{x}^{2}+D_{y}^{2}\right) v+i \zeta\left[1+A\left(D_{x}^{2}+D_{y}^{2}\right)\right]\right\} P_{f}^{n} .
\end{aligned}
$$

Unfortunately, using equation (20) directly to solve for $P_{f}^{n+1}$ presents a problem because of the $\left(D_{x}^{2}+D_{y}^{2}\right)$ term. 
Discretizing the $x$ - and $y$-axes and approximating the differential operators with difference operators results in a system of $N^{2}$ simultaneous equations, where $N$ is the number of grid points in either the $x$ or $y$ direction. Even with the most efficient solution algorithms, processing this system of equations requires a computational effort proportional to $N^{3}$. In addition, these operations must be performed for each frequency component at each depth step, a procedure far too costly for most practical applications.

The traditional method to circumvent this problem is to approximate equation (20) with a system in which the operators that depend on $D_{x}^{2}$ have been split from those which depend on $D_{y}^{2}$. We follow a similar approach which is outlined below.

First let us rewrite equation (20) as

$$
\begin{aligned}
& (1-i \zeta)\left[1+\gamma_{-}\left(D_{x}^{2}+D_{y}^{2}\right) v+i \delta_{-}\left(D_{x}^{2}+D_{y}^{2}\right)\right] P_{f}^{n+1} \\
& =(1+i \zeta)\left[1+\gamma_{+}\left(D_{x}^{2}+D_{y}^{2}\right) \tau+i \delta_{+}\left(D_{x}^{2}+D_{y}^{2}\right)\right] P_{f}^{n},
\end{aligned}
$$

where

$$
\gamma_{ \pm}=(1 \pm i \zeta)^{-1} \frac{1}{r} B
$$

and

$$
\delta_{ \pm}= \pm(1 \pm i \zeta)^{-1} \zeta A .
$$

Now we use the following approximation:

$$
\begin{aligned}
{[1+} & \left.\gamma\left(D_{x}^{2}+D_{y}^{2}\right) v+i \delta\left(D_{x}^{2}+D_{y}^{2}\right)\right] \\
\approx & {\left[1+\gamma\left(D_{x}^{2}+D_{y}^{2}\right) v+i \delta\left(D_{x}^{2}+D_{y}^{2}\right)\right] } \\
& +\left(\gamma D_{x}^{2} v+i \delta D_{x}^{2}\right)\left(\gamma D_{y}^{2} v+i \delta D_{y}^{2}\right) \\
= & {\left[1+\gamma D_{x}^{2} v+i \delta D_{x}^{2}\right]\left[1+\gamma D_{y}^{2} v+i \delta D_{y}^{2}\right] . }
\end{aligned}
$$

Note that the error term $\left(\gamma D_{x}^{2} v+i \delta D_{x}^{2}\right)\left(\gamma D_{y}^{2} v+i \delta D_{y}^{2}\right)$ is nearly zero (zero in the homogeneous case) for energy propagating along either the $x$ or $y$ axis and is a maximum for energy propagating along a $45^{\circ}$ rotation of these axes. Putting relation (22) into equation (21), we have the following system:

$$
\begin{aligned}
(1-i \zeta)\left[1+\gamma_{-} D_{x}^{2} v+i \delta_{-} D_{x}^{2}\right] \\
\quad \times\left[1+\gamma_{-} D_{y}^{2} v+i \delta_{-} D_{y}^{2}\right] P_{f}^{n+1} \\
=(1+i \zeta)\left[1+\gamma_{+} D_{x}^{2} v+i \delta_{+} D_{x}^{2}\right] \\
\quad \times\left[1+\gamma_{+} D_{y}^{2} v+i \delta_{+} D_{y}^{2}\right] P_{f}^{n},
\end{aligned}
$$

or, rewriting, we obtain

$$
\begin{aligned}
& {\left[(1-i \zeta)+\frac{1}{v} B D_{x}^{2} v-i \zeta A D_{x}^{2}\right](1-i \zeta)^{-1}} \\
& \quad \times\left[(1-i \zeta)+\frac{1}{v} B D_{y}^{2} v-i \zeta A D_{y}^{2}\right] P_{f}^{n+1} \\
& =\left[(1+i \zeta)+\frac{1}{v} B D_{x}^{2} v+i \zeta A D_{x}^{2}\right](1+i \zeta)^{-1}
\end{aligned}
$$

$$
\times\left[(1+i \zeta)+\frac{1}{v} B D_{y}^{2} v+i \zeta A D_{y}^{2}\right] P_{f}^{n} .
$$

In the remainder of this discussion, the system described by equation (20) is referred to as the unsplit-operator system and that given by equation (23) as the split-operator system.

The advantage of using the split-operator system is that the computational effort needed to solve these equations is now proportional to $N^{2}$. The solution of this system is obtained by using the following recursion:

$$
\begin{aligned}
A_{0} b_{1} & =b_{0}, \\
b_{2} & =A_{1} b_{1},
\end{aligned}
$$

and

$$
A_{2} P_{j}^{n+1}=b_{2},
$$

where

$$
\begin{aligned}
b_{0}= & {\left[(1+i \zeta)+\frac{1}{v} B D_{x}^{2} v+i \zeta A D_{x}^{2}\right](1+i \zeta)^{-1} } \\
& \times\left[(1+i \zeta)+\frac{1}{v} B D_{y}^{2} v+i \zeta A D_{y}^{2}\right] P_{f}^{n},
\end{aligned}
$$

$$
\begin{aligned}
& A_{0}=\left[(1-i \zeta)+\frac{1}{v} B D_{x}^{2} \bar{z}-i \zeta A D_{x}^{2}\right], \\
& A_{1}=(1-i \zeta),
\end{aligned}
$$

and

$$
A_{2}=\left[(1-i \zeta)+\frac{1}{v} B D_{y}^{2} v-i \zeta A D_{y}^{2}\right] .
$$

Discretizing the $x$ - and $y$-axes and using second-order finitedifference approximations for $D_{x}^{2}$ and $D_{y}^{2}$ reduces equations (24a) and (24c) into tridiagonal matrix systems for which solutions are easily generated (e.g., Claerbout, 1985, p. 98).

Using these equations, the computational sequence to extrapolate the wave field one step in $z$ would proceed as follows: (1) calculation of $b_{0}$ for all points in the model plane $\left(x_{i}, y_{j}, z=z_{n}\right)[i=1, \ldots, n x ; j=1, \ldots, n y]$, (2) application of equation (24a) along strips of $x$ for each point $y_{i}$, (3) application of equation (24b) for all points $\left(x_{i}, y_{j}\right)$, and (4) application of equation (24c) along strips of $y$ for each point $x_{i}$ of the model plane.

It should also be noted that the implementation of the above steps is performed easily in a parallel or vector processing environment. This is trivial for steps (1) and (3). For step (2), note that the application of equation (24a) is independent of $y$ in the sense that the solution along the $x$ strip at $y_{j}$ does not depend on the solution at $y_{j-1}, y_{j+1}$ or at any other value of $y$. Likewise, in step (4) the application of equation (24c) is independent of the variable $x$. Thus, equation (24a) can be solved for all points $y_{j}$ in parallel and equation (24c) solved for all points $x_{i}$ in parallel.

\section{Accuracy of the split-operator system}

The problem with the split-operator system [equation (23)], as mentioned earlier, is that its accuracy varies azi- 
muthally. One way to analyze this variation is to examine the phase difference between the unsplit-operator system and the split-operator system as a function of azimuth. In order to perform the analysis, we restrict ourselves to a homogeneous medium and then transform the equations into the wavenumber-frequency domain. The details of this process are presented in Appendix A. From equations (A-2) and (A-4) the two systems can be written as

$\hat{P}_{f}^{n+1}=\exp (i \phi) \exp \left(i \phi_{0}\right) \hat{P}_{f}^{n} \quad$ (unsplit system)

and

$\hat{P}_{f}^{n+1}=\exp (i \phi) \exp \left(i \phi_{x}\right) \exp \left(i \phi_{y}\right) \hat{P}_{f}^{n} \quad$ (split system),

where

$$
\begin{array}{rlrl}
\phi & =2 \tan ^{-1}(\zeta), \\
\phi_{0} & =2 \tan ^{-1}\left(a_{0}\right), & & a_{0}=\delta\left(K_{x}^{2}+K_{y}^{2}\right) /\left[1+\gamma\left(K_{x}^{2}+K_{y}^{2}\right)\right], \\
\phi_{x} & =2 \tan ^{-1}\left(a_{1}\right), & a_{1}=\delta K_{x}^{2} /\left(1+\gamma K_{x}^{2}\right),
\end{array}
$$

and

$\phi_{y}=2 \tan ^{-1}\left(a_{2}\right), \quad a_{2}=\delta K_{r}^{2} /\left(1+\gamma K_{y}^{2}\right)$,

with $\gamma+i \delta=-(B+i \zeta A) /(1+i \zeta), \hat{P}_{f}^{n}=\hat{P}_{f}\left(k_{x}, k_{y}, z=z_{n}\right.$; $\omega)$. and the wavenumbers are represented as $\left(\gamma^{2} / \omega^{2}\right) k_{x}^{2}=K_{x}^{2}$ and $\left(r^{2} / \omega^{2}\right) k_{y}^{2}=K_{y}^{2}$.

Comparing equations (25), we write the phase difference between the two systems as

$$
\phi_{d}=\phi_{0}-\phi_{x}-\phi_{y} .
$$

In Figure 1, curve A plots the phase difference between the unsplit operator (25a) and the split operator (25b) as a function of propagation angle for an azimuth of $45^{\circ}\left(K_{x}=\right.$ $K_{y}$ ). Note that there is a significant phase difference between the two for propagation angles beyond $30^{\circ}$. For this comparison we have chosen $A=0.855$ and $B=0.355$.

\section{The phase-correction filter}

If we can derive a filter with a phase operator given by equation (27) and can apply it to the system (23) at each extrapolation step, we can effectively remove the azimuthal anisotropy of the extrapolation system. In order to derive the desired filter, let us define

$$
\begin{aligned}
\phi_{d} & =\tan ^{-1}\left(a_{3}\right), \\
a_{3} & =\frac{\operatorname{Im}(F)}{\operatorname{Re}(F)},
\end{aligned}
$$

where $F$ is the filter that we desire. Approximating $\tan ^{-1}\left(a_{i}\right) \approx a_{i}$ in equations (26) and (28) and then using these equations in equation (27), we can write

$$
a_{3} \approx-2 \gamma \delta K_{x}^{2} K_{y}^{2}\left\{\frac{\left[2+\gamma\left(K_{x}^{2}+K_{y}^{2}\right)\right]}{\left(1+\gamma K_{x}^{2}\right)\left(1+\gamma K_{y}^{2}\right)\left[1+\gamma\left(K_{x}^{2}+K_{y}^{2}\right)\right]}\right\} .
$$

This approximation is appropriate for $\left(K_{x}^{2}+K_{y}^{2}\right)<1$, since $a_{0}, a_{1}$, and $a_{2} \sim \xi$ in this region and $\zeta \ll 1$. The first-order approximation gives us

$$
F=1-i 4 \gamma \delta K_{. x}^{2} K_{y}^{2} .
$$

Applying the phase-correction filter of equation (30) to the split-operator system does a good job in reducing the anisotropy of the extrapolation operator as demonstrated in Figure 1, curve B. This plot is the same as curve A, except that the phase of the split operator has been modified by the phase correction filter. Even with the first-order approximation, the phase difference is less than one percent for propagation angles out to $45^{\circ}$.

Taking higher order terms in $a_{3}$ gives a better match to the phase of the original operator; however, taking higher order terms also increases the cost to implement the system. As it turns out, there is a much simpler way to achieve better accuracy. By redefining $F$ as

$$
F=1-i 4 \varepsilon_{0} \gamma \delta K_{x}^{2} K_{y}^{2} .
$$

where $\varepsilon_{0}$ is an adjustable parameter, we can extend the accuracy of this filter to cover a wider range of propagation angles. This increased accuracy is shown in Figure 1, curve C. Here we have the same phase comparison as before except that we have used the filter given by equation (31) with $\varepsilon_{0}=1.5$. In this case, the phase difference is less than one percent for propagation angles out to and beyond $60^{\circ}$.

\section{Stability considerations}

Using the phase-correction filter as defined by equation (31). in general, results in an unstable extrapolation system,

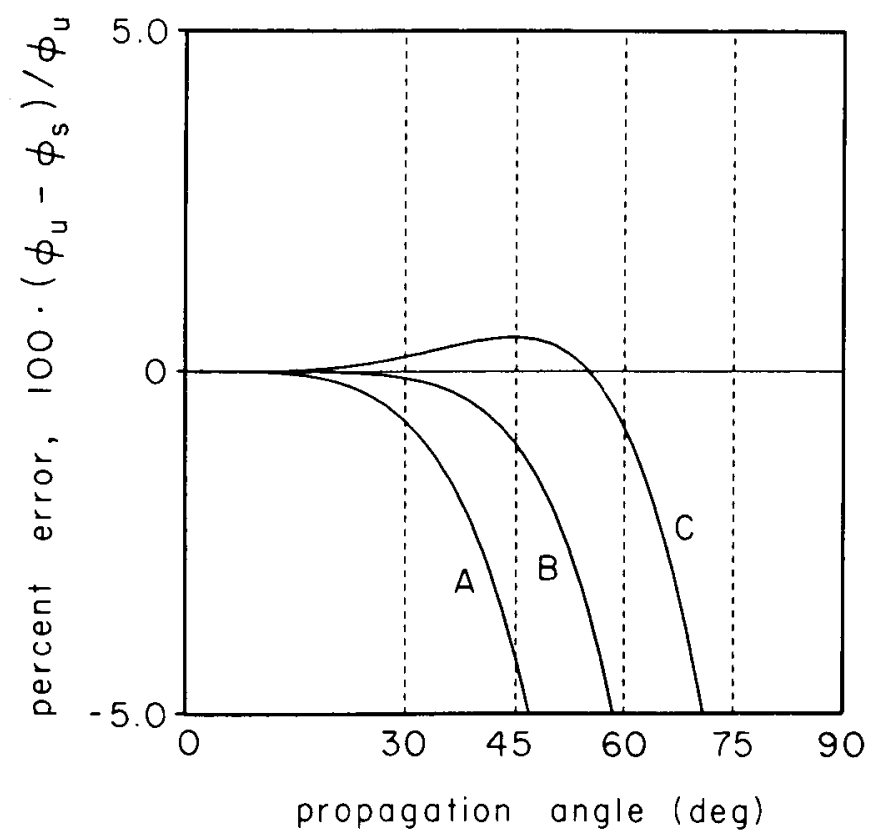

FIG. 1. Phase difference between the unsplit $\left(\phi_{u}\right)$ and split $\left(\phi_{s}\right)$ extrapolation systems at an azimuth of $45^{\circ}$. We have set $\zeta=0.125$ for these calculations, although the results are fairly insensitive to variations in $\omega, \Delta z$, and $\%$. Curve A shows the phase difference calculated with no correction term. Curve B shows the phase difference calculated with application of the first-order correction term [equation (30)]. Curve $C$ shows the phase difference calculated with the correction term and $\varepsilon_{0}=1.5$ [equation (31)]. 
because the magnitude of this filter is greater than unity for all nonzero values of $K_{x}^{2}$ and $K_{y}^{2}$. To compensate for this effect, a damping function must be added to the filter. We have found that a filter of the form

$$
F=D_{1}\left[1-i 4 \varepsilon_{0} D_{2} \gamma \delta K_{x}^{2} K_{y}^{2}\right]
$$

with

$$
D_{1}=\left(1+\varepsilon_{1} K_{x}^{4}\right)^{-1}\left(1+\varepsilon_{1} K_{y}^{4}\right)^{-1}
$$

and

$$
D_{2}=\left(1+\varepsilon_{2} K_{x}^{4}\right)^{-1}\left(1+\varepsilon_{2} K_{y}^{4}\right)^{-1}
$$

works very well. This filter is stable for

$$
\varepsilon_{2} \geq \frac{1}{\varepsilon_{1}} \varepsilon_{0}^{2} \gamma^{2} \delta^{2}-\frac{3}{8} \varepsilon_{1}
$$

The use of two damping terms may seem like an unnecessary complication; however, it allows us to choose the values of $\varepsilon_{1}$ and $\varepsilon_{2}$ such that the magnitude of the operator is near unity for all $\left(K_{x}^{2}+K_{y}^{2}\right) \leq 1$. Using filter (32) with $\varepsilon_{1} \approx 0.01$ is most effective. Larger values of $\varepsilon_{1}$ provide too much damping and deteriorate the signal in the region $\left(K_{x}^{2}+\right.$ $\left.K_{y}^{2}\right) \leq 1$; smaller values of $\varepsilon_{1}$ require too large a value of $\varepsilon_{2}$ to be used and thus significantly decrease the effectiveness of the filter. Finally, it should be noted that the addition of the damping terms has no effect on the phase of the filter.

As shown in Appendix B, we can implement filter (32) in the spatial domain as a cascade of tridiagonal matrix systems. We will symbolically represent this operation as

$$
Q_{f}^{n+1}=\mathscr{F} P_{f}^{n+1}
$$

where $\mathscr{F}$ is the spatial domain operator corresponding to filter (32), $P_{f}^{n+1}$ is the extrapolated wave field calculated from equations (24), and $Q_{f}^{n+1}$ is the phase-corrected wave field.

For laterally varying media, we simply let $v \rightarrow v(x, y)$ in the filter 厈. Although this substitution neglects the issue of operator ordering, it is still appropriate, since the phasecorrection filter is only a first-order correction to the extrapolation system. Further modifications to the filter result, in general, in a more complicated system to implement.

Applying this operator to the extrapolation process produces excellent results. Here, we compare calculations performed with and without the phase-correction operator. In both cases, the output is viewed as a time slice on a plane located 20 grid points away from the source point (Figure 2). The geometry of the model is such that at this time the energy arriving in this plane is propagating at an angle of about $60^{\circ}$ with respect to the extrapolation direction. The first panel (Figure 3a) was computed without the phasecorrection operator and exhibits a characteristic diamond shape indicative of the azimuthal variation in accuracy which is inherent to the split-operator system. The second calculation (Figure 3b) included the application of the phasecorrection operator to the wave field at each step in the extrapolation process. Note that the wave field in this panel is essentially circular and shows no apparent azimuthal variations.
Dip filtering

The extrapolation operators derived from the continued fraction expansion are designed to match the exact dispersion relation best for $\left(K_{x}^{2}+K_{y}^{2}\right)<1$. However, in the evanescent region of $k_{z}\left[\right.$ [i.e., $\left(K_{x}^{2}+K_{y}^{2}\right)>1$ ] these operators do a very poor job of matching the exact operator. In fact, for both 2-D and 3-D calculations, the operators can produce significant artifacts from energy propagating in this region as illustrated in Figure 4, which shows the numerical point response of the extrapolation system presented earlier. In this example, the output is viewed as a time slice in the $x z$ plane at $y=0$, with the source located at the origin. No filtering has been applied to this calculation. The proper response for this projection is a quarter-circle, and it is

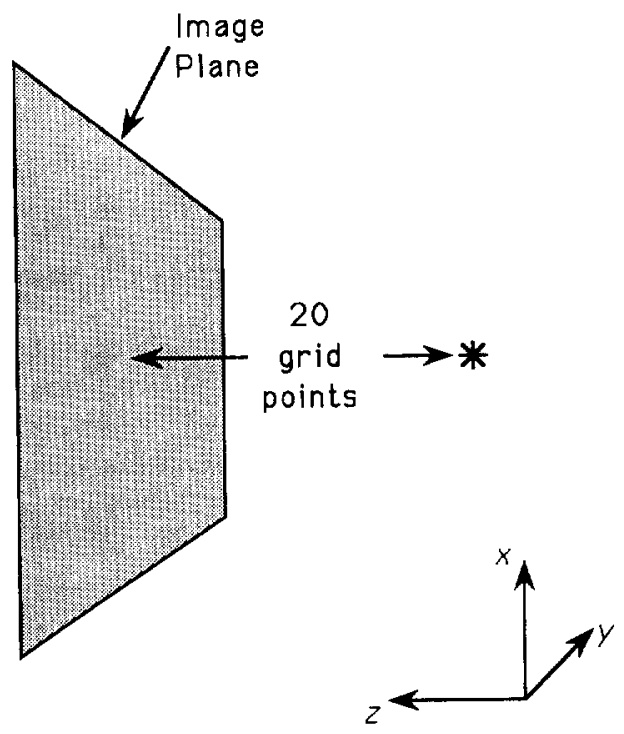

FIG. 2. Model geometry for phase-correction filter comparison. (a)

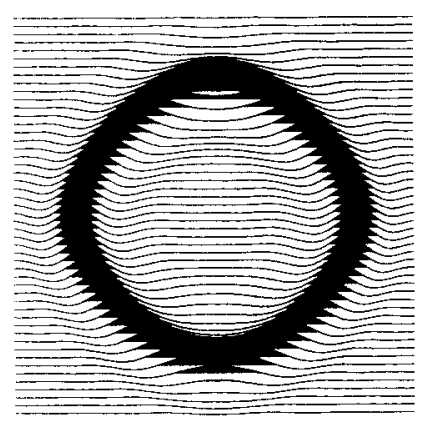

(b)

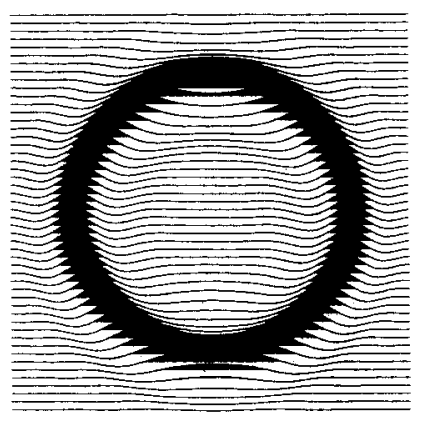

Fig. 3. Phase-correction filter comparison. (a) 3-D splitoperator extrapolation result calculated without application of the phase-correction filter. The azimuthal variation in accuracy is characteristic of the split-operator system. (b) Same calculation as (a) except the phase-correction operator has been applied to the propagation system at each extrapolation step. Note the nearly circular wavefront resulting from the filtering prócess. 
evident from Figure 4 that the paraxial solution does well for propagating angles out to about $60^{\circ}$. Beyond this point, the solution becomes less accurate with increasing dip and the wavefront actually curls back under itself, producing a secondary arrival. This secondary cusp-like arrival is an artifact arising from energy propagating in the region $\left(K_{x}^{2}+\right.$ $\left.K_{y}^{2}\right)>1$. Although the secondary wavefront has a slower group velocity than the main wavefront, it can produce arrivals which interfere with energy of interest and results in a solution which is both confusing and difficult to interpret. In order to prevent this situation from occurring, the energy in this region needs to be suppressed.

Fortunately, suppression can be realized using the same filtering technique described in the previous section. Each of the damping terms in the phase-correction filter (32) also acts as a dip filter which strongly suppresses energy in the region $\left(K_{x}^{2}+K_{y}^{2}\right)>1$. This behavior can be seen by examining the general form of the damping terms given by

$$
\begin{gathered}
\hat{Q}_{f}=D \hat{P}_{f}, \\
D=\left(1+\varepsilon K_{x}^{2 n}\right)^{-1}\left(1+\varepsilon K_{y}^{2 n}\right)^{-1}, \quad n=1,2, \cdots
\end{gathered}
$$

with $\varepsilon \ll 1$. In the region $\left|K_{x}\right| \ll 1$ or $\left|K_{y}\right| \ll 1, D \rightarrow 1$ (all pass); and in the region $\left|K_{x}\right| \gg 1$ or $\left|K_{y}\right| \gg 1, D \rightarrow 0$ (reject). The parameter $n$ controls the sharpness of the cutoff between the pass and reject zones.

To demonstrate the effectiveness of the dip filter, we have recalculated the point response and applied relation (34) with $\varepsilon_{1}=0.01$ and $n=2$ at each depth step. The result is shown in Figure 5. Note that a large portion of the energy in the evanescent zone has been eliminated, while energy in the region $\left(K_{x}^{2}+K_{y}^{2}\right)<1$ has not been visibly affected.

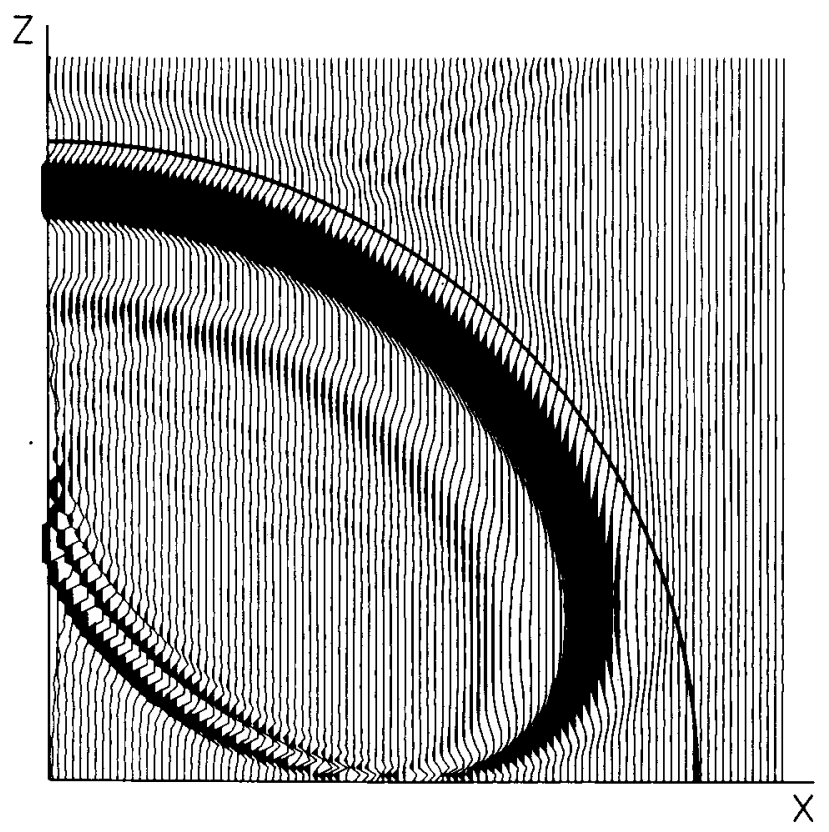

FIG. 4. Time slice in the $x z$ plane showing the point response of the $60^{\circ}$ paraxial system. Proper response for this projection is the quarter circle denoted by the heavy curve. The secondary cusp-like waveform near the origin is due to energy propagating in the region $\left(K_{x}^{2}+K_{y}^{2}\right)>1$.

\section{Boundary conditions}

We consider three types of boundary conditions to be applied along the edges of the model grid: (1) zero value (to represent a free surface), (2) zero slope (to represent a plane of symmetry), and (3) absorbing (to represent an infinite medium). Since the paraxial system is first order along the $z$-axis (extrapolation direction), any of the above conditions can be specified exactly for boundaries perpendicular to this axis. For the $x$ and $y$ dimensions, the boundary conditions must be applied when solving the tridiagonal matrix systems at each extrapolation step. In this case, exact representations of conditions (1) and (2) are readily prescribed and a very good approximate absorbing condition is given by the B3 formulation of Clayton and Engquist (1980).

\section{THE SCATTERING MATRIX}

The scattering matrix forms the coupling between the forward-scattered and backscattered waves in the presence of heterogeneous media. Incorporating these effects within the extrapolation system [equation (10)], we have the following relation:

$$
\partial \cdot\left[\begin{array}{l}
P_{f} \\
P_{b}
\end{array}\right]=\left[\begin{array}{rr}
i \alpha & 0 \\
0 & -i \alpha
\end{array}\right]\left[\begin{array}{l}
P_{f} \\
P_{b}
\end{array}\right]+\mathbf{S}\left[\begin{array}{l}
P_{f} \\
P_{b}
\end{array}\right],
$$

where

$$
\mathbf{S}=\frac{s}{\Delta z}\left[\begin{array}{rr}
-1 & 1 \\
1 & -1
\end{array}\right]
$$

and

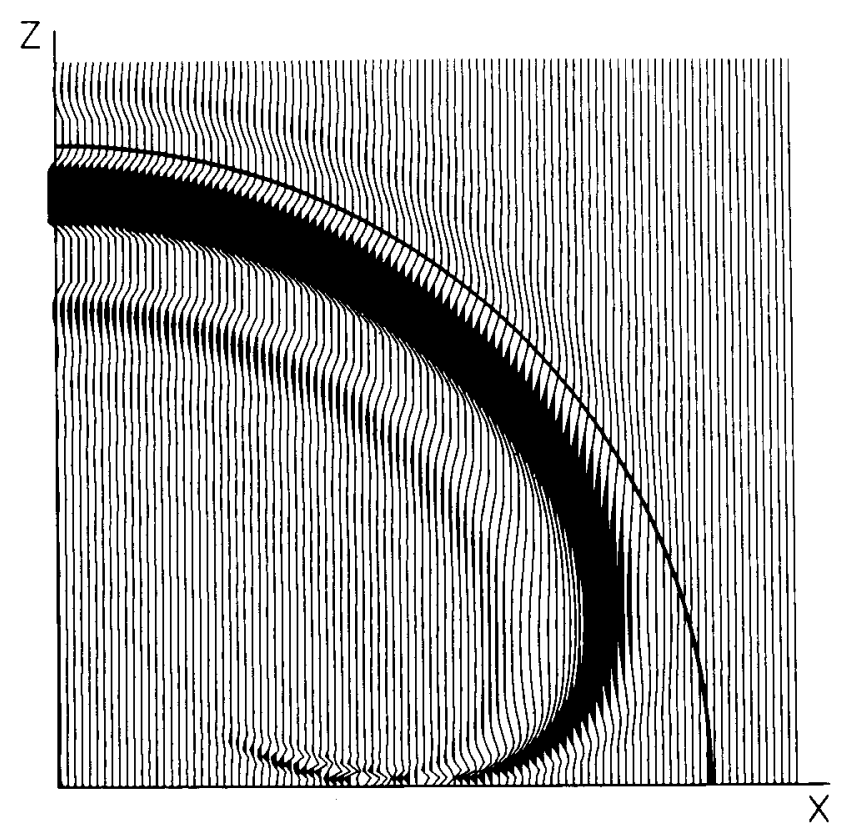

FIG. 5. Same calculation as Figure 4 except dip filter operator has been applied at each extrapolation step reducing the energy in the evanescent zone significantly. 


$$
s=\frac{\Delta z}{2}\left[\frac{1}{\alpha} \rho\right]\left[\begin{array}{l}
\frac{1}{\rho} \alpha \\
\rho
\end{array}\right] \text {. }
$$

as determined from equations (7) and (9). The subscript $z$ in the above equation refers to differentiation of that term with respect to the variable $z$. Writing the system (36) as a set of coupled difference equations, we get

$$
\begin{aligned}
P_{f}^{n+1}-P_{f}^{n}= & i \phi\left(P_{f}^{n+1}+P_{f}^{n}\right) \\
& +\frac{s}{2}\left[\left(P_{f}^{n+1}+P_{f}^{n}\right)-\left(P_{b}^{n+1}+P_{b}^{n}\right)\right]
\end{aligned}
$$

and

$$
\begin{aligned}
P_{b}^{n+1}-P_{b}^{n}= & -i \phi\left(P_{b}^{n+1}+P_{b}^{n}\right) \\
& -\frac{s}{2}\left[\left(P_{f}^{n+1}+P_{f}^{n}\right)-\left(P_{b}^{n+1}+P_{b}^{n}\right)\right],
\end{aligned}
$$

where $\phi=\alpha \Delta z / 2$.

Using these equations to extrapolate an incident forwardscattered wave field $P_{f}^{n}$ from depth step $z_{n}$ to depth step $z_{n+1}$, we would expect to generate a transmitted (forwardscattered) wave field $P_{f}^{n+1}$ at step $z_{n+1}$ and a reflected (backscattered) wave field $P_{b}^{n}$ at step $z_{n}$. In addition, since there is no incident backscattered field, we can set $P_{b}^{n+1}=0$. Solving for the unknown fields $P_{b}^{n}$ and $P_{f}^{n+1}$, we have

$$
P_{b}^{n}=\left[\frac{s}{1+\phi^{2}}\right] P_{f}^{\prime}
$$

and

$$
P_{f}^{n+1}=\left[1+\frac{s}{1+\phi^{2}}\right] P_{f}^{\prime}
$$

where

$$
P_{f}^{\prime}=\frac{(1+i \phi)}{(1-i \phi)} P_{f}^{n}
$$

In deriving these relations, we have neglected terms arising from the operator $\alpha$ acting on $s$ and the operator $s$ acting on itself.

Examining equations (39), we see that the operator $s /\left(1+\phi^{2}\right)$ is analogous to a reflection coefficient. When $s=0$ (homogeneous medium), these relations reduce to

$$
P_{b}=0
$$

and

$$
P_{f}^{n+1}=\frac{(1+i \phi)}{(1-i \phi)} P_{f}^{n}
$$

the second of which is simply the difference form of the propagation system given by equation (11). When $s$ is nonzero, $P_{f}^{\prime}$ is first calculated from equation (40) using the techniques described in the previous section; then $P_{b}^{n}$ and $P_{f}^{n+1}$ can be computed from equations (39a) and (39b).

In order to implement these equations, we must again use a suitable approximation for $\alpha$. The zeroth order approximation $\left(\alpha_{0}=\omega / v\right)$ yields the following:

$$
\frac{s}{1+\phi^{2}} \approx \frac{1}{\left(1+\zeta^{2}\right)} \frac{\Delta z}{2}\left(\frac{\rho_{z}}{\rho}+\frac{\nu_{z}}{y}\right), \quad \zeta=\frac{\omega \Delta z}{2 y},
$$

with $\rho_{z}=\partial \rho / \partial z$ and $y_{z}=\partial v / \partial z$. This expression is exact for normally incident plane waves in a layered medium. Higher order formulations can be developed by using more accurate representations for $\alpha$. These expressions tend to be mathematically cumbersome and are not presented here. In the examples presented in this paper, we have used the planewave approximation for the scattering term. Since we are mainly interested in modeling problems in which most of the energy is traveling along the propagation axis, the zeroth order approximation is probably adequate for these cases.

\section{SOURCE EXCITATION}

To initialize the extrapolation process, we can specify either a force or pressure source to be used directly in equation (10) or, alternatively, we can specify an entire wave field along one $x-y$ plane of the model space. By convention, this plane is designated as $\left(x, y, z=z_{0}\right)$, with the initial wave field denoted as $P_{j}^{0}=P_{f}\left(x, y, z=z_{0}, \omega\right)$. We prefer the latter method, since it is much more versatile than the first.

In general, the wave field $P_{f}^{0}$ may be determined by numerical or analytical techniques, or it may be specified by an observed wave field, as is the case with migration. This flexibility allows the extrapolation method to be coupled with a wide variety of other wave-propagation techniques.

In the 2-D and 3-D examples shown in this paper, we have used an analytic expression to represent the initial wave field $P_{f}^{0}$. The source location may be set inside or outside the actual computational grid and the analytic response due to the source is then calculated for all grid points in the plane $\left(x, y, z=z_{0}\right)$.

For 3-D calculations, the initial pressure field is given by

$$
\begin{aligned}
P_{f}^{0} & =S(\omega) \frac{1}{r} \exp (i \omega r / v), \\
r & =\left[\left(x-x_{s}\right)^{2}+\left(y-y_{s}\right)^{2}+\left(z_{0}-z_{s}\right)^{2}\right]^{1 / 2} .
\end{aligned}
$$

For 2-D calculations, the exact line source representation is given in the frequency domain by the Hankel function of order zero. Using the asymptotic or far-field approximation to the Hankel function, we have

$$
\begin{gathered}
P_{f}^{0}=S(\omega) \sqrt{i /(\omega r)} \exp (i \omega r / v), \\
r=\left[\left(x-x_{s}\right)^{2}+\left(z-z_{s}\right)^{2}\right]^{1 / 2} .
\end{gathered}
$$

In each of the above equations, $S(\omega)$ is the temporal Fourier transform of the source time function and the source is located at $\left(x_{s}, y_{s}, z_{s}\right)$.

For display purposes, we would like to make the 2-D calculations appear as if they were initiated with a point source so that we can compare 2-D and 3-D computations more accurately. Equations (44) and (43) differ only by a factor of $\sqrt{-i \omega / r}$, representing the difference in geometric spreading between the line source and point source. Although we cannot account for this spreading difference explicitly within the calculations, the 2-D solutions can be modified after the fact by multiplication with ań additional factor of $\sqrt{-i \omega / r}$, a process 
that is only approximate, since we do not know the exact length $r$ of the travel path for a given arrival.

\section{IMPLEMENTATION CONSIDERATIONS}

\section{Computational attributes}

A major advantage of using the first-order extrapolation system given by equation (10) is that the system requires knowledge of the wave field only at the previous depth step in order to calculate the solution at the present depth step. Computationally, this means that 2-D problems can be solved by stepping through the model with the solution being calculated only along a $1-\mathrm{D}$ vector; likewise, 3-D problems can be solved by extrapolation of the solution on a 2-D plane. This procedure effectively reduces active storage requirements by one dimension compared to complete wavesolution techniques. Thus, large 2-D and even realistic 3-D problems can be handled by existing computers.

By casting the extrapolation system in the frequency domain, we have the opportunity to apply weighting functions to the individual frequency components while the solution is being calculated. Frequency-domain representation allows for the implementation of various types of filters to the wave field and also allows us to model attenuation and viscoelastic effects as an arbitrary function of frequency.

Another advantage of formulating the extrapolation equations in the frequency domain is that solutions can be calculated for each frequency component independently, making the system highly suitable for implementation on parallel or vector processing computers. In this type of configuration, the solutions for a number of different frequencies can be generated simultaneously on separate processors or in sequence on a vector processor.

\section{Time slices and movies from a limited number of frequencies}

The production of time slices and movies is quite efficient for the paraxial operators because they are cast in the frequency domain. The trick is to arrange for the source to emit a source pulse periodically. If, for display purposes, a source wavelet that is a single cycle of a sinusoid is adequate, representing the source requires just a small number of frequencies.

To demonstrate this, consider a simple pulsating source function. The source emits a sinusoidal wavelet of width $\tau$ every $n \tau$ seconds. The initial pulse is shifted $a$ seconds from $t=0$. A mathematical description of this source is given by

$$
s(t)= \begin{cases}\exp [i 2 \pi(t-a) / \tau+i \phi], & \text { if } t_{0}-\tau / 2 \leq t \leq t_{0}+\tau / 2 \\ 0, & \text { otherwise }\end{cases}
$$

where $t_{0}=(\ell n \tau)+a ;(\ell=-\infty, \ldots,-1,0,1, \ldots, \infty)$, and $\phi$ is the initial phase of the source. The periodicity of the source makes it a candidate for a Fourier series representation

$$
s(t)=\sum_{k=-\infty}^{x} c_{k} \exp \left[i 2 \pi \frac{k t}{n \tau}\right]
$$

Solving for the $c_{k}$, we have

$$
c_{k}=\frac{1}{n} \exp \left[i \phi-i 2 \pi \frac{a k}{n \tau}\right] \operatorname{sinc}[\pi(1-k / n)]
$$

where sinc $(x)$ is the usual $\sin (x) / x$ function. A reasonable representation of the source can be achieved with only the $2 n-1$ frequencies (Fourier coefficients $k=1, \ldots, 2 n-1$ ) that sample the central peak of the sinc function as demonstrated in Figure 6a, where we have set $n=4$ and computed $s(t)$ for various numbers of frequencies. Note that a very good result is obtained for as few as seven frequencies. Increasing the value of $n$ makes the time separation between successive pulses greater (Figure 6b).

To make time slices then, the solution is summed with the weights given by equation (47). That is.

$$
\text { slice }(x, y, z)=\sum_{k=1}^{2 n-1} c_{k}(a, \phi, n) P_{j}\left(x, y, z, \omega=\frac{2 \pi k}{n \tau}\right) \text {. }
$$

The multiple time slices for a movie are constructed by simply marching the shift factor $a$ from 0 to $n \tau$, at which point the film loop repeats. This process is demonstrated in Figure 6c.

\section{EXAMPLES}

\section{2-D example: Fault-block model}

In this example, we compare results from the paraxial extrapolator (PE) technique with a conventional time-domain FD calculation for the simple fault-block model shown in Figure 7. For this comparison, the source time function is given by the first derivative of a Gaussian pulse, i.e.,

$$
s(t)=t \exp \left(-t^{2} / a^{2}\right),
$$
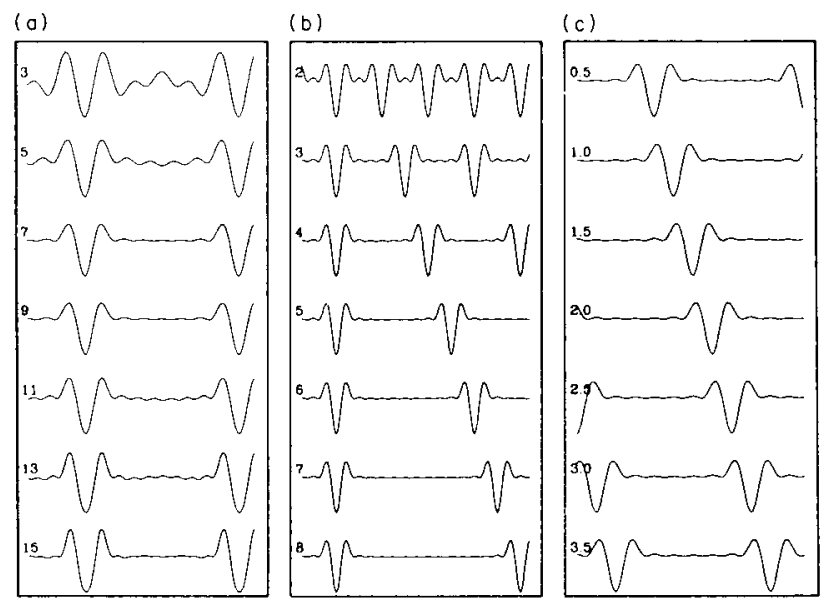

FIG. 6. Representation of periodic source time function. (a) Source representation for $n=4$ computed by summation for the number of frequencies shown to the left of each trace. (b) Source representation for the value of $n$ shown to the left of each trace. (c) Movie panels obtained by variation of the shift factor $a$ from $0.5 \tau$ to $3.5 \tau[n=4, n w=7]$. 


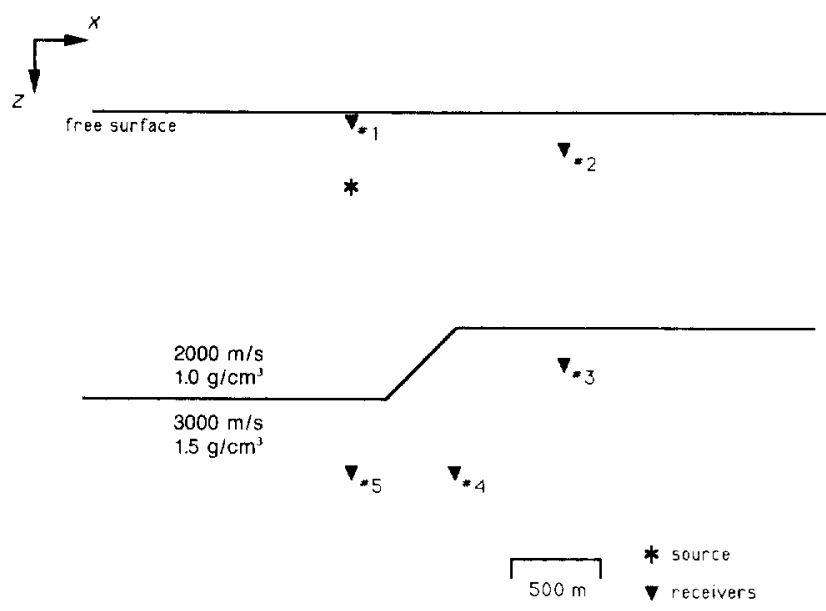

FIG. 7. Fault-block model showing media variations as well as source and receiver locations. where we have set $a=0.15 \mathrm{~s}$. The source amplitude spectrum peaks at about $15 \mathrm{~Hz}$ and has significant power out to $45 \mathrm{~Hz}$. The grid spacing in the PE calculation is $5 \mathrm{~m}$ laterally and $2.5 \mathrm{~m}$ vertically and in the FD model, the grid spacing is $5 \mathrm{~m}$. The output is viewed as both time-slice snapshots (Figure 8) and seismograms recorded at the locations indicated in Figure 7 (Figure 9). First we discuss the time-slice comparison.

Figure 8 shows wave-field snapshots at four selected times for both the FD and PE results. Note that the PE result is split into two columns. The first shows the downgoing forward-scattered wave from the source, while the second shows the direct upgoing wave from the source, as well as the first-order backscattered wave from the structural interface. Higher order scattering effects (i.e., multiples) are not included in the PE time-slice computation. In addition, it should be noted that the PE results have been corrected to mimic a point-source calculation and the FD results have not been so modified. In general, the agreement between the two calculations is quite good. Some of the discrepancies are due
FD
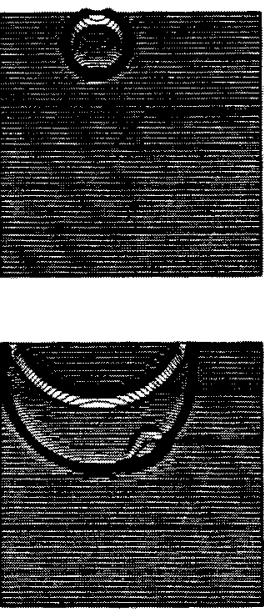

PE

forward sweep
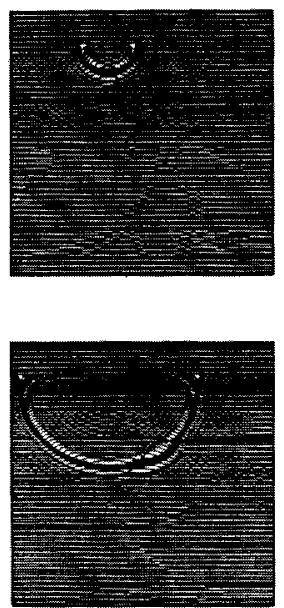

$i=0.44$
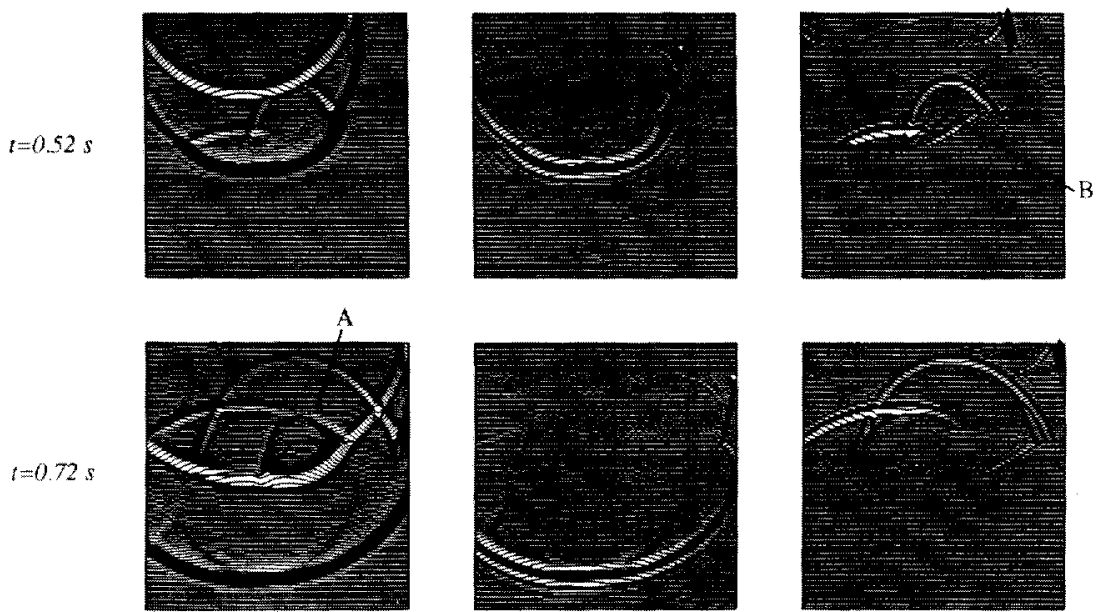

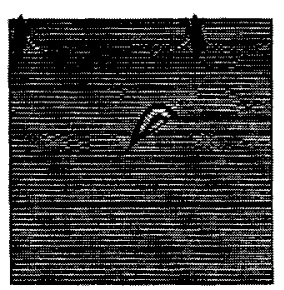

PE

reverse sweep
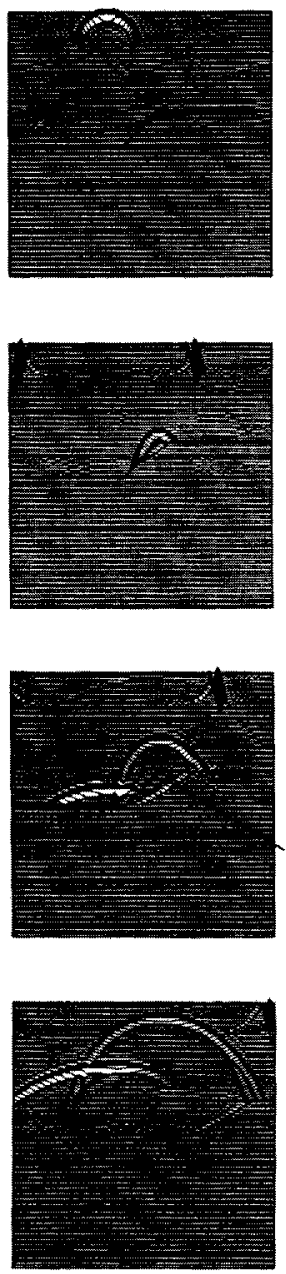

FIG. 8. Time-slice comparison for fault-block model. FD result (left panel), PE forward sweep result (middle panel), and $P E$ reverse sweep result (right panel). 
to the omission of the higher order scattering effects in the $P E$ results (e.g., the wavefront labeled $A$ at $t=0.72 \mathrm{~s}$ in the $F D$ result is not present in the $P E$ calculation). Other differences can be attributed to artifacts arising from the presence of evanescent energy in the PE calculation (e.g., the wavefront labeled $\mathrm{B}$ at $t=0.52 \mathrm{~s}$ in the reverse sweep of the $\mathrm{PE}$ result). These artifacts are usually not significant and in most cases can be easily identified.

Comparing the seismograms in Figure 9, we again see a very good overall agreement between the two techniques. In particular, the results from the two methods computed at receiver locations nos. 1, 4, and 5 agree very well with one another in timing, phase, and amplitude. Note that for this comparison, both sets of results have been transformed to mimic point-source calculations. At receiver location no. 2, the $\mathrm{PE}$ result does not do so well in matching the arrivals predicted by the FD result because most of the energy arriving at this location is propagating at about $85^{\circ}$ with respect to the extrapolation direction. Since the paraxial approximation is only accurate out to $60^{\circ}$, we would not expect it to model this energy correctly. As indicated in this comparison, waves which are propagating at angles outside the range of validity of the paraxial approximation are still modeled, although they travel at a group velocity which is slower than the correct value. If we were interested in modeling this energy more accurately, we could rotate the $\mathrm{PE}$ grid by $90^{\circ}$ and then extrapolate the solution horizontally. The arrival labeled $\mathrm{A}$ on this record corresponds to the

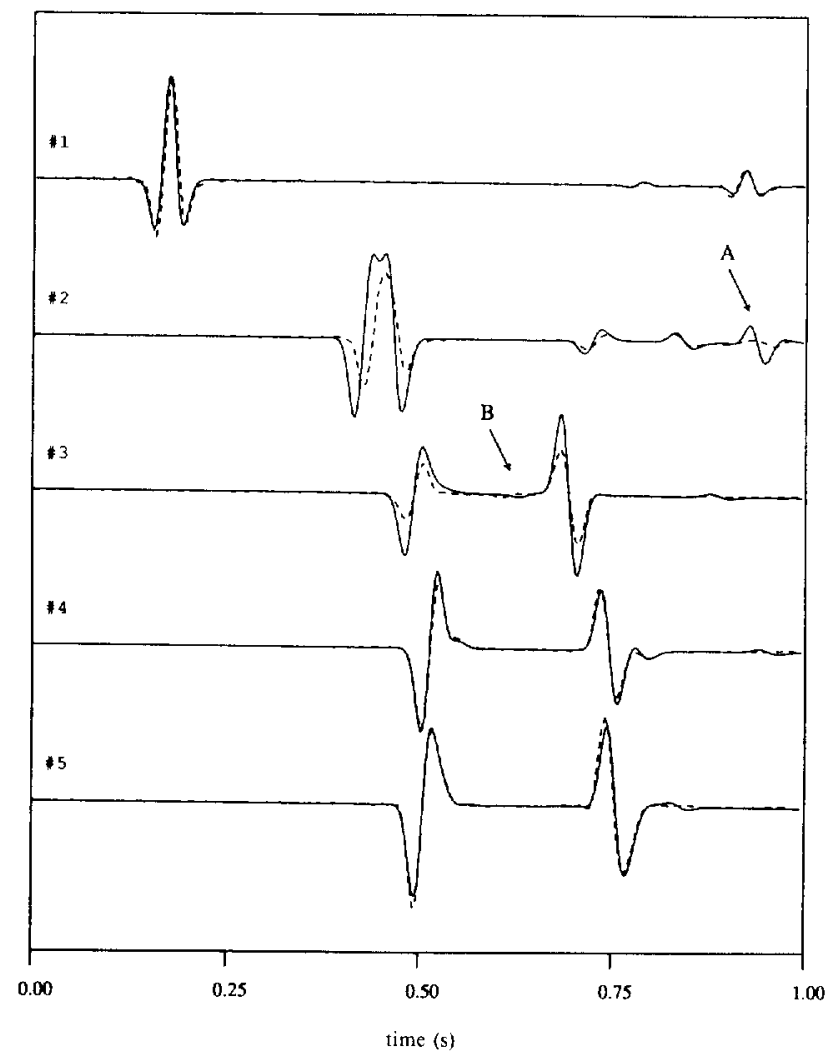

FIG. 9. Seismogram comparison for fault-block model. Solid line is FD result and dashed line is PE result. See Figure 7 for receiver positions. wavefront $\mathrm{A}$ in Figure 8. Moving on to the result at location no. 3 , we see that the timing and phase of the two calculations agree quite well; however, the amplitude of the PE result is significantly smaller than that predicted by the FD calculation. Energy arriving at this station propagates across the overlying layer boundary with an incidence angle between $35^{\circ}$ and $40^{\circ}$. Since the velocity and density increase at this interface, the transmission coefficient grows as the angle of incidence is increased. However, as discussed previously, we have approximated the transmission coefficient with its value at normal incidence and, consequently, we have underestimated the strength of the transmitted energy computed in the $\mathrm{PE}$ result. The arrival labeled $\mathrm{B}$ on this record corresponds to the artifact $\mathrm{B}$ in Figure 8 . Note that this arrival has very little energy and does not interfere with the rest of the signal.

\section{3-D example: Crustal basin}

Due to a variety of constraints, 2-D models are often used as approximations for structures which are known to be 3-D. This can be a problem because it is often difficult to fully understand the effects that 3-D structure will have on seismic wave propagation.

This effect of modeling 3-D behavior with a 2-D model is illustrated in the following example, where we show a comparison between solutions generated for a 3-D model and a 2-D approximation to this model. The model consists of a simple low-velocity basin situated in a higher velocity background medium (Figure 10). Along the top of the model we have imposed a free-surface boundary condition and all other model boundaries are absorbing. Note that the 2-D model is obtained by taking a vertical cross-section of the 3-D model along the strike of the survey line ( $x z$ plane, Figure 10). For the 2-D model, we have computed the result using both conventional finite-differences and the PE technique. The grid spacing for the PE calculations is $10 \mathrm{~m}$ in the $x$ and $y$ directions and $5 \mathrm{~m}$ in the $z$ direction. In the $\mathrm{FD}$ model, the grid spacing is constant at $10 \mathrm{~m}$. The source time function is the same as in the previous example, except here

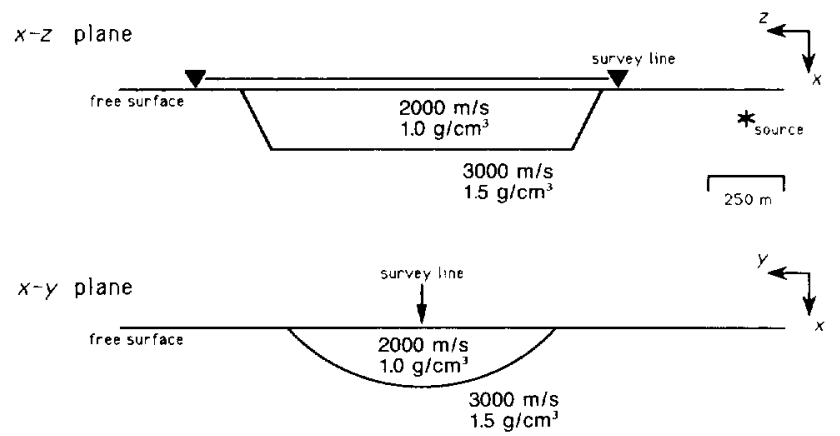

FIG. 10. Model geometry and media parameters for 2-D versus 3 -D comparison. Top panel shows $x z$ plane along strike of the survey line. Source location is within this plane. In addition, this slice represents the model used in the 2-D calculations. Bottom panel shows cross-line model structure for 3-D calculation. 
we have $a=0.2 \mathrm{~s}$. The amplitude spectrum of this source peaks at about $12 \mathrm{~Hz}$.

The solutions for both models are displayed as time sections recorded at regular intervals along the survey line (Figure 11). In the PE calculations we have included only contributions from the direct forward-scattered energy for a single pass of the extrapolator through the model. Thus, the far-end backscattered basin reflections seen in the FD result are not present in either of the PE results. In addition, we have accounted for geometric spreading differences by multiplying the results of the 2-D calculation by a scale factor of $\sqrt{-i \omega / r}$, where $r$ is the distance between the source and receiver. The amplitudes in each solution have been normalized to the nearest offset trace.

In this comparison, we focus our attention on the modeling of the multiply reflected and refracted waves occurring within the basin structure. First, examining the 2-D model results, we see that aside from the omission of the backscattered energy, the PE solution agrees very well with the result obtained using the FD technique. Comparing the 2-D results

2D basin model (FD)

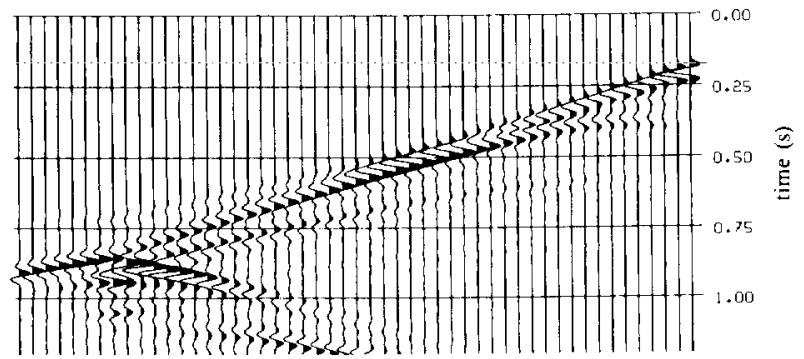

2D basin model (PE)

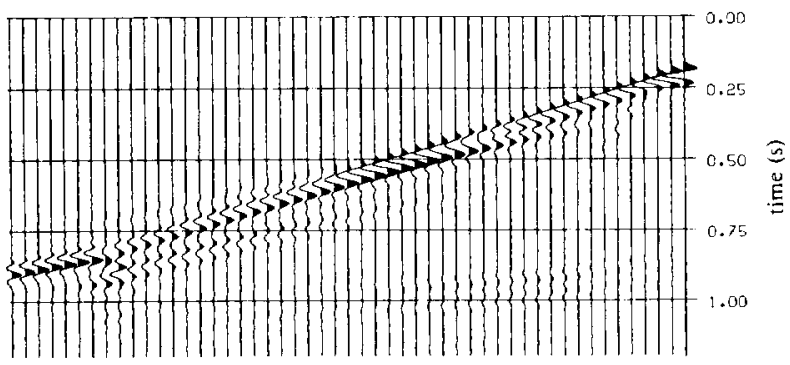

$3 \mathrm{D}$ basin model (PE)

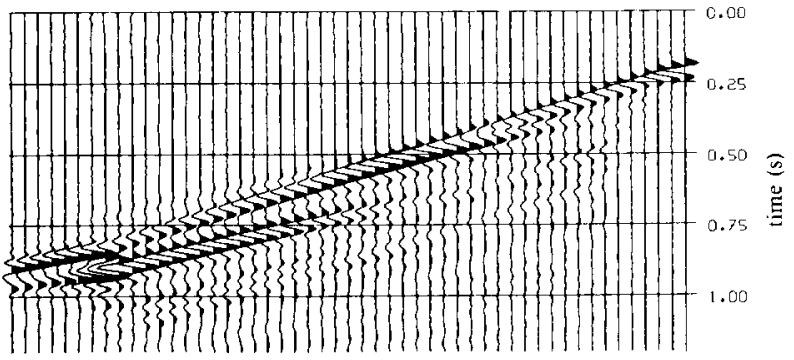

FIG. 11. Time sections along survey line for basin model comparison. 2-D FD result (top panel), 2-D PE result (middle panel), and 3-D PE result (bottom panel). with the 3-D result, we find that in many respects the $2-\mathrm{D}$ solutions are quite similar to the results of the 3-D calculation. The timing and phase for many of the arrivals within the basin agree well between the two models. For these considerations, the 2-D model may well be an appropriate substitute for the 3-D structure. However, the 2-D model clearly fails to model correctly the amplitudes of the arrivals within the basin. In fact, the 3-D solution shows arrivals which have more than twice the amplitude of the corresponding arrivals in the 2-D sections. In addition, the coda of the 3-D records is much more complicated than that seen in the $2-\mathrm{D}$ results, indicating the presence of cross-line structure. This strong focusing of energy in the 3-D calculation is caused by the sloping edges of the basin floor. Although the dip of these boundaries is not extreme, their effects are quite significant.

Unfortunately there is no way to account for this scattering phenomenon in the 2-D calculation. In order to produce similar results using a 2-D model, we would need to alter the media parameters and possibly change the geometry of the basin as well. Either of these choices would result in an incorrect interpretation of the structure for this experiment.

\section{CONCLUSIONS}

In this-paper we have presented an approximate numerical technique in which acoustic waves are modeled using a system of paraxial extrapolators. The technique is developed by casting the equations of motion into the frequency domain and then formulating them as a first-order spatial extrapolation system. This approach is attractive because it requires significantly less computer memory as compared to more complete methods such as conventional time-domain finite-difference calculations. In fact, when using the paraxial method for large 2-D and 3-D problems, the model size is determined primarily by the time required to perform the necessary computations rather than being restricted by memory availability and/or accessibility.

To obtain an estimate of the computational requirements of the paraxial method, we consider the following comparison. Performing an explicit fourth-order time-domain finitedifference calculation of a constant-density 3-D model requires on the order of 50 floating point operations (fpo) per grid point per time step. For the same model, one pass of the paraxial method (including the phase correction operator) requires about 500 fpo per grid point per frequency. However, the number of time steps needed for the finite-difference calculation is typically an order of magnitude greater than the number of frequencies needed for the paraxial calculation. From this, we conclude that both methods generally require the same order of operations to compute a given solution.

Obviously, to model problems in which the effects of higher order scattering are important requires more than one pass with the paraxial technique; however, the cost of added passes increases only linearly with the number of passes performed. Furthermore, the explicit separation of the wave field into its forward-scattered and backscattered components as provided by the paraxial method is desirable and, in fact, sometimes necessary in the study of many wavepropagation problems. 


\section{ACKNOWLEDGMENTS}

We would like to thank Amoco Foundation Inc. for the generous fellowship support of R. W. G. during this study. Some of the computations were done on a Convex $\mathrm{Cl} / \mathrm{XP}$ purchased under NSF grant EAR-8721205. Cindy Arvesen assisted with the drafting of figures.

Contribution 4746, Division of Geological and Planetary Sciences, California Institute of Technology, Pasadena, California.

\section{REFERENCES}

Claerbout, J. F., 1970, Coarse grid calculations of waves in inhomogeneous media with application to delineation of complicated seismic structure: Geophysics, 35, 407-418.

- 1985, Imaging the earth's interior: Blackwell Scientific Publ. Clayton, R. W., and Engquist, B., 1977, Absorbing boundary conditions for acoustic and elastic wave equations: Bull., Seis. Soc. Am., 67, 1529-1540.

- 1980. Absorbing side boundary conditions for wave-equation migration: Geophysics, 45, 895-904.

Fricke, J. R., 1988, Reverse-time migration in parallel: A tutorial: Geophysics, 53, 1143-1150.

Hale, D., and Claerbout, J. F., 1983, Butterworth dip filters: Geophysics, 48, 1033-1038.

Halpern, L., and Trefethen, L. N., 1988, Wide-angle one-way wave equations: J. Acoust. Soc. Am., 84, 1397-1404.

Kelly, K. R., Ward, R. W., Treitel, S., and Alford, R. M., 1976, Synthetic seismograms-A finite-difference approach: Geophysics, 41, 2-27.

Kosloff, D. D., and Baysal, E., 1982, Forward modeling by a Fourier method: Geophysics, 47, 1402-1412.

Marfurt, K. J.. 1984, Accuracy of finite-difference and finite-element modeling of the scalar and elastic wave equations: Geophysics, 49. $533-549$

Reshef, M., Kosloff, D., Edwards, M.. and Hsiung, C., 1988a, Three-dimensional acoustic modeling by the Fourier method: Geophysics, 53, 1175-1183.

1988b, Three-dimensional elastic modeling by the Fourier method: Geophysics, 53, 1184-1193.

\section{APPENDIX A}

\section{WAVENUMBER-DOMAIN REPRESENTATION OF THE EXTRAPOLATION EQUATIONS}

We begin with the split-operator system as defined by equation (21)

$$
\begin{aligned}
& (1-i \zeta)\left[1+\gamma_{-}\left(D_{x}^{2}+D_{y}^{2}\right) v+i \delta_{-}\left(D_{x}^{2}+D_{y}^{2}\right)\right] P_{f}^{n+1} \\
& \quad=(1+i \zeta)\left[1+\gamma_{+}\left(D_{x}^{2}+D_{y}^{2}\right) \vartheta+i \delta_{+}\left(D_{x}^{2}+D_{y}^{2}\right)\right] P_{f}^{n} .
\end{aligned}
$$

Assuming a homogeneous medium, we can Fourier transform this equation into the wavenumber domain to obtain

$$
\hat{P}_{f}^{n+1}=\frac{(1+i \zeta)\left[1+(\gamma+i \delta)\left(K_{x}^{2}+K_{y}^{2}\right)\right]}{(1-i \zeta)\left[1+(\gamma-i \delta)\left(K_{x}^{2}+K_{y}^{2}\right)\right]} \hat{P}_{f}^{n},
$$

where $\gamma \pm i \delta=-(B \pm i \zeta A) /(1 \pm i \zeta), \hat{P}_{f}^{n}=\hat{P}_{f}\left(k_{x}, k_{y}, z=z_{n}\right.$, $\omega)$, and we have the Fourier transform pairs

$$
D_{x}^{2}=\frac{y^{2}}{\omega^{2}} \partial_{x x} \leftrightarrow \frac{y^{2}}{\omega^{2}} k_{x}^{2}=K_{x}^{2}
$$

and

$$
D_{y}^{2}=\frac{v^{2}}{\omega^{2}} \partial_{y y} \leftrightarrow \frac{y^{2}}{\omega^{2}} k_{y}^{2}=K_{y}^{2} .
$$

Equation (A-2) is the wavenumber-domain representation of the unsplit operator system. Now using the following approximation

$$
\begin{aligned}
& {\left[1+(\gamma+i \delta)\left(K_{x}^{2}+K_{y}^{2}\right)\right]} \\
& \quad \approx\left[1+(\gamma+i \delta)\left(K_{x}^{2}+K_{y}^{2}\right)\right]+(\gamma+i \delta)^{2} K_{x}^{2} K_{y}^{2} \\
& \quad=\left[1+(\gamma-i \delta) K_{x}^{2}\right]\left[1+(\gamma-i \delta) K_{y}^{2}\right],
\end{aligned}
$$

we can derive the wavenumber-domain representation of the split-operator system from equation (A-2). This representation is given by

$\hat{P}_{f}^{n+1}=\frac{(1+i \zeta)\left[1+(\gamma+i \delta) K_{x}^{2}\right]\left[1+(\gamma+i \delta) K_{y}^{2}\right]}{(1-i \zeta)\left[1+(\gamma-i \delta) K_{x}^{2}\right]\left[1+(\gamma-i \delta) K_{y}^{2}\right]} \hat{P}_{f}^{n}$.

\section{APPENDIX B}

\section{SPATIAL-DOMAIN IMPLEMENTATION OF THE PHASE CORRECTION FILTER}

We begin with the phase correction operation defined in the wavenumber-frequency domain by

$$
\hat{Q}=D_{1}\left[1-i 4 \varepsilon_{0} D_{2} \gamma \delta K_{x}^{2} K_{y}^{2}\right] \hat{P}
$$

with

$$
D_{1}=\left(1+\varepsilon_{1} K_{x}^{4}\right)^{-1}\left(1+\varepsilon_{1} K_{y}^{4}\right)^{-1}
$$

and

$$
D_{2}=\left(1+\varepsilon_{2} K_{r}^{4}\right)^{-1}\left(1+\varepsilon_{2} K_{y}^{4}\right)^{-1},
$$

where $\hat{P}\left(k_{x}, k_{y}, z, \omega\right)$ is the input wave field and $\hat{Q}\left(k_{x}, k_{y}, z, \omega\right)$ is the filtered output. Letting $\hat{P}^{\prime}=$ $-i 4 \varepsilon_{0} \gamma \delta K_{r}^{2} K_{y}^{2} \hat{P}$, we can rewrite equation (B-1) as

$$
\hat{Q}=D_{1}\left[\hat{P}+D_{2} \hat{P}^{\prime}\right]
$$

Now, the solution to this system can be built up by considering equations which have the following general form:

$\hat{Q}_{\text {out }}=D \hat{Q}_{\text {in }}$,

$$
D=\left(1+\varepsilon K_{x}^{2 n}\right)^{-1}\left(1+\varepsilon K_{y}^{2 n}\right)^{-1}, \quad n=1,2, \ldots
$$

where $n=2$ for the operators $D_{1}$ and $D_{2}$ in equation (B-2).

Following the method outlined by Hale and Claerbout (1983), we can write equation (B-3) as a cascade of secondorder filters given by 


$$
\hat{Q}_{\text {out }}=\left(\prod_{j=0}^{n-1} \frac{\eta_{j}}{\eta_{j}-K_{x}^{2}} \prod_{k=0}^{n-1} \frac{v_{k}}{v_{k}-K_{y}^{2}}\right) \hat{Q}_{\mathrm{in}},
$$

where

$$
\eta_{i}=\varepsilon^{-1 / n} \exp \left[i(2 j+1) \frac{\pi}{n}\right]
$$

and

$$
v_{k}=\varepsilon^{-1 / n} \exp \left[i(2 k+1) \frac{\pi}{n}\right] .
$$

Applying each term of the products in equation (B-4) in a recursive fashion, we obtain the following sequence of second-order systems:

$$
\begin{aligned}
\hat{Q}_{v_{0}} & =\left[\frac{v_{0}}{v_{0}-K_{y}^{2}}\right] \hat{Q}_{\text {in }}, \\
\hat{Q}_{\nu_{1}} & =\left[\frac{v_{1}}{\nu_{1}-K_{y}^{2}}\right] \hat{Q}_{v_{0}}, \\
\hat{Q}_{v_{n-1}} & =\left[\frac{v_{n-1}}{v_{n-1}-K_{y}^{2}}\right] \hat{Q}_{v_{n-2}}, \\
\hat{Q}_{\eta_{0}} & =\left[\frac{\eta_{0}}{\eta_{0}-K_{x}^{2}}\right] \hat{Q}_{\nu_{n-1}} \\
\hat{Q}_{\eta_{1}} & =\left[\frac{\eta_{1}}{\eta_{1}-K_{x}^{2}}\right] \hat{Q}_{\eta_{0}} \\
\hat{Q}_{\text {out }} & =\left[\frac{\vdots}{\eta_{n-1}-K_{x}^{2}}\right] \hat{Q}_{\eta_{n-2}} .
\end{aligned}
$$

Clearing the denominators and transforming each of these to the spatial domain, we obtain the following series of equations:

$$
\begin{aligned}
& {\left[1+v_{0}^{-1} \frac{v^{2}}{\omega^{2}} \partial_{y y}\right] Q_{v_{0}}=Q_{\text {in }},} \\
& {\left[1+v_{1}^{-1} \frac{v^{2}}{\omega^{2}} \partial_{y y}\right] Q_{v_{1}}=Q_{v_{0}}}
\end{aligned}
$$

$$
\begin{aligned}
& {\left[1+v_{n-1}^{-1} \frac{v^{2}}{\omega^{2}} \partial_{y y}\right] Q_{v_{n-1}}=Q_{v_{n-2}},} \\
& {\left[1+\eta_{0}^{-1} \frac{v^{2}}{\omega^{2}} \partial_{x x}\right] Q_{\eta_{0}}=Q_{v_{n-1}},} \\
& {\left[1+\eta_{1}^{-1} \frac{v^{2}}{\omega^{2}} \partial_{x x}\right] Q_{\eta_{1}}=Q_{\eta_{0}},} \\
& \text { ! } \\
& {\left[1+\eta_{n-1}^{-1} \frac{v^{2}}{\omega^{2}} \partial_{x x}\right] Q_{\text {out }}=Q_{\eta_{n-2}} .}
\end{aligned}
$$

Upon discretization of the $x$ - and $y$-axes, these equations can be implemented as a sequence of tridiagonal matrix systems.

This sequence of equations can be written symbolically in the following manner:

$$
Q_{\text {out }}=\underset{\sim}{\mathbf{D}} Q_{\text {in }},
$$

where $\mathbf{D}$ is the matrix operator which represents the operations performed in equations (B-7). Using this notation, the phase-correction filter can then be represented in the spatial domain as

$$
Q=\underline{\mathbf{D}}_{1}\left[\boldsymbol{P}+\underline{\mathbf{D}}_{2} \boldsymbol{P}^{\prime}\right],
$$

where $P(x, y, z, \omega)$ is the input wave field, $P^{\prime}=-i 4 \varepsilon_{0} \gamma \delta\left(v^{2} / \omega^{2}\right) \partial_{x x}\left(v^{2} / \omega^{2}\right) \partial_{y y} P$, and $Q(x, y, z, \omega)$ is the filtered output. 